\title{
Approaches to quality improvement in nursing homes: Lessons learned from the six-state pilot of CMS's Nursing Home Quality Initiative
}

\author{
Stephanie Kissam*1, David Gifford ${ }^{1,2}$, Peggy Parks ${ }^{\dagger 3}$, Gail Patry ${ }^{1}$, \\ Laura Palmer $^{\dagger 4}$, Linda Wilkes ${ }^{\dagger 5}$, Matthew Fitzgerald ${ }^{\dagger 6}$, \\ Alice Stollenwerk Petrulis ${ }^{\dagger 7}$ and Leslie Barnette ${ }^{\dagger 8}$
}

\begin{abstract}
Address: ${ }^{1}$ Rhode Island Quality Partners, Providence RI, 02908, USA, ${ }^{2}$ Division of Geriatrics, Brown University, Providence RI, 02912, USA ${ }^{3}$ Centers for Medicare \& Medicaid Services, Baltimore MD, USA, ${ }^{4}$ Colorado Foundation for Medical Care, Aurora CO, 80014, USA, ${ }^{5} \mathrm{Florida}$ Medical Quality Assurance, Tampa FL, 33607, USA, ${ }^{6}$ Delmarva Foundation, Easton MD, 21601, USA, 7 Ohio KePRO, Seven Hills OH, 44131, USA and ${ }^{8}$ Qualis Health, Seattle WA, 98133, USA

Email: Stephanie Kissam* - skissam.ripro@sdps.org; David Gifford - David_Gifford@brown.edu; Gail Patry - gpatry.ripro@sdps.org; Laura Palmer - lpalmer.copro@sdps.org; Linda Wilkes - lwilkes.flpro@sdps.org; Matthew Fitzgerald - mfitzgerald@dfmc.org; Alice Stollenwerk Petrulis - apetrulis.ohpro@sdps.org; Leslie Barnette - lesb@qualishealth.org

* Corresponding author †Equal contributors
\end{abstract}

Published: 16 May 2003

BMC Geriatrics 2003, 3:2

This article is available from: http://www.biomedcentral.com/I47|-23/8/3/2

(c) 2003 Kissam et al; licensee BioMed Central Ltd. This is an Open Access article: verbatim copying and redistribution of this article are permitted in all media for any purpose, provided this notice is preserved along with the article's original URL.
Received: 17 January 2003

Accepted: 16 May 2003

\begin{abstract}
Background: In November 2002, the Centers for Medicare \& Medicaid Services (CMS) launched a Nursing Home Quality Initiative that included publicly reporting a set of Quality Measures for all nursing homes in the country, and providing quality improvement assistance to nursing homes nationwide. A pilot of this initiative occurred in six states for six months prior to the launch.
\end{abstract}

Methods: Review and analysis of the lessons learned from the six Quality Improvement Organizations (QIOs) that led quality improvement efforts in nursing homes from the six pilot states.

Results: QIOs in the six pilot states found several key outcomes of the Nursing Home Quality Initiative that help to maximize the potential of public reporting to leverage effective improvement in nursing home quality of care. First, public reporting focuses the attention of all stakeholders in the nursing home industry on achieving good quality outcomes on a defined set of measures, and creates an incentive for partnership formation. Second, publicly reported quality measures motivate nursing home providers to improve in certain key clinical areas, and in particular to seek out new ways of changing processes of care, such as engaging physicians and the medical director more directly. Third, the lessons learned by QIOs in the pilot of this Initiative indicate that certain approaches to providing quality improvement assistance are key to guiding nursing home providers' desire and enthusiasm to improve towards a using a systematic approach to quality improvement.

Conclusion: The Nursing Home Quality Initiative has already demonstrated the potential of public reporting to foster collaboration and coordination among nursing home stakeholders and to heighten interest of nursing homes in quality improvement techniques. The lessons learned from this pilot project have implications for any organizations or individuals planning quality improvement projects in the nursing home setting. 


\section{Background}

In November 2001, the Centers for Medicare \& Medicaid Services (CMS) announced plans for the Nursing Home Quality Initiative, a new strategy to improve the quality of care in the nation's nursing homes. This Initiative is a four-pronged effort. First, CMS and state survey and certification agencies continue their existing regulatory enforcement efforts. Second, CMS promotes consumers' access to new information about the quality of care in specific nursing homes, in the form of publicly-reported Quality Measures available on CMS's website for consumers, http://www.medicare.gov. Third, nursing home stakeholders collaborate to promote and support efforts to improve nursing home quality of care. Fourth, Quality Improvement Organizations (QIOs) offer communitybased quality improvement assistance programs to nursing home providers seeking to improve their performance on the Quality Measures.

Before national implementation of the Nursing Home Quality Initiative in November 2002, CMS conducted a pilot in six states: Colorado, Florida, Maryland, Ohio, Rhode Island, and Washington. QIO quality improvement activities during the pilot included collaborating with nursing home stakeholders on all aspects of the initiative, educating all nursing home providers on the Quality Measures, and offering quality improvement assistance to nursing home providers. The Nursing Home Quality Initiative pilot built on nursing home providers' desire and enthusiasm to deliver high quality care by adding another incentive to improve (the public release of Quality Measure data) and by providing consultative services, clinical education, and technical support from QIOs to aide in that improvement.

Releasing performance outcome information (Quality Measures data) gives consumers additional information to consider when selecting a nursing home, and consequently, may also spur efforts to improve the quality of care provided in nursing homes overall [1]. These efforts to improve quality may result from increased consumer consciousness due to public reporting, and, consequently, heightened competition in the nursing home market based on relative quality of care, or may be a response to the incentive to compare favourably with other individual facilities based on publicly-reported data [2,3]. There may remain other motivating factors, but still open to debate is which effect has the greatest impact on healthcare providers' efforts to improve [4].

In a report on the appropriateness of the Quality Measures chosen for publicly reporting during the Nursing Home Quality Initiative pilot in six states, the General Accounting Office (GAO) questioned the reliability, validity, and risk adjustment of the Quality Measures selected for public reporting, expressed concerns about the accuracy of data that are used to calculate the Quality Measures, and indicated that the format in which Quality Measure data were published on Nursing Home Compare may not be understandable by consumers [5]. However, these potential limitations do not seem to have reduced the impact of public reporting on nursing home providers' interest in quality improvement and QIO assistance, as experienced by the QIOs in the pilot states. Furthermore, according to CMS's own data, 52\% of nursing homes in the six pilot states requested technical assistance on quality improvement from the QIOs, and most nursing homes in these six states $(88 \%)$ had heard of the Nursing Home Quality Initiative to publicly report quality of care information [6].

This paper reports the lessons that CMS and the six pilot states learned with regard to the impact that this Initiative has on nursing home providers' interest in quality improvement, on the relationship among stakeholders in the nursing home setting, and on the provision of quality improvement assistance to nursing homes. We present recommendations based on these lessons learned in order to inform and facilitate future quality improvement efforts in nursing homes.

Table I: Recommended Approaches to Quality Improvement in the Nursing Home Setting

\begin{tabular}{ll}
\hline Approaches & Form partnerships with nursing home stakeholders \\
- & Establish relationship with State Survey Agency (SSA) \\
- & Promote the use of quality measures in quality improvement \\
- & Engage physicians and medical directors in quality improvement \\
- & Seach principles of quality improvement to all nursing home staff, including direct care staff \\
- & Provide one-on-one assistance to nursing homes \\
- & Convert the regulatory compliance culture to a quality improvement culture \\
- & Address high rates of staff turnover and position vacancies in nursing homes \\
\hline
\end{tabular}




\section{Methods}

QIOs serve as external facilitators of quality improvement projects among Medicare providers. In the pilot, QIOs provided quality improvement assistance related to two of the clinical topics covered by the publicly reported Quality Measures, pain management and pressure ulcer prevention and treatment. These two topics were selected from the entire set of publicly reported measures because nursing home providers regard improvement in these areas as a high priority, and because the QIOs in the pilot states wanted to test and refine methods and materials for quality improvement assistance on just two topics before developing them for all topics associated with the publicly reported Quality Measures. QIOs convened volunteer nursing home providers to facilitate the exchange of ideas and strategies among providers for improving care processes related to these two clinical topics, as well as visited nursing homes to work with providers on an individual basis to provide quality improvement assistance. Additionally, QIOs provided to these nursing homes clinical materials and educational workshops on pain management and pressure ulcer prevention and treatment, as well as education on quality improvement strategies such as team building, brainstorming, root-cause analysis, and rapid-cycle improvement. While we can identify the types of assistance that QIOs provided to participating nursing homes, we did not have a tracking system in place during the pilot to quantify the amount of assistance provided by state, and each QIO utilized a unique combination of these strategies to assist nursing home providers in its state. Therefore, the activities as they are described here should be viewed as representative examples of approaches used in nursing home quality improvement.

Throughout the pilot, the QIOs in the six pilot states met on weekly one-hour teleconference calls to share lessons learned about each stage of implementing the pilot. During these calls, staff at Rhode Island Quality Partners (the QIO for Rhode Island and the lead QIO for the pilot) collected lessons learned and comments from QIOs, national trade organizations, and CMS. The lessons learned were entered into an Access database and categorized by state, category of intervention (such as partnerships, recruitment, workshops, quality improvement assistance, etc.) and clinical topic (e.g., pain, pressure ulcers, restraints, etc.). The authors reviewed the database entries and analysed the recorded lessons learned to identify common themes in order to summarize the following experiencebased recommended approaches to assisting nursing home providers with quality improvement.

\section{Results}

Table 1 summarizes the recommended approaches to conducting quality improvement projects in the nursing home setting.

\section{Forming Partnerships}

In order to raise awareness about CMS's Nursing Home Quality Initiative among nursing home providers and consumers, nursing home stakeholders in the six pilot states found it effective to work together in promoting the availability of nursing home data for consumers in the form of Quality Measures, as well as to guide the improvement activities related to the Quality Measures. Stakeholders in each state included nursing home trade associations (the state affiliates of the American Health Care Association and the American Association of Homes and Services for the Aged), nursing home professional organizations (such as the American College of Health Care Administrators and the American Association of Nurse Assessment Coordinators), state agencies (such as the Departments of Health, Survey and Certification, and Elderly Affairs), long-term care ombudsman offices, hospital discharge planners, Resident Council representatives, and senior citizen advocacy groups. Based on the experiences of the pilot states, the recommended approaches to facilitating partnerships are to: 1) form an advisory or steering committee of partners that meets regularly (e.g., monthly), and 2) maintain regular contact with stakeholders, even when no new information is available.

Benefits of creating a steering committee of nursing home stakeholders include: easing communication among all partners, facilitating updates, discussing issues facing nursing homes, and brainstorming possible solutions. For this reason, nursing home stakeholders in each state expressed a preference to work together, even in states where there has traditionally been tension between certain organizations. Partnerships result in practical coordination of resources, such as scheduling training sessions to nursing homes at pre-existing conferences sponsored by other partners, or sharing address lists, newsletters, and meeting space. Such partnerships also allow for the development of a consistent, positive message about nursing home issues to all outside parties. For example, it was noted in the pilot that when the media received a consistent message about the Nursing Home Quality Initiative from all stakeholders, media coverage tended to be more supportive of nursing homes than when stakeholders were divided.

Since frequent contact with all nursing home stakeholders increases trust and collaboration among the different groups, timely communication with all potential partners is a recommended approach to building partnerships. Several QIOs in the pilot sent out a weekly newsletter by email to all stakeholders. This newsletter conveyed information about upcoming training sessions, legislative updates and answers to frequently asked questions. 
Table 2: Recommended Approaches to Engaging Physicians and Medical Directors in Quality Improvement

\begin{tabular}{ll}
\hline Approaches implemented by nursing home providers & $\begin{array}{l}\text { Identify a physician champion in the nursing home that could involve other physicians in improv- } \\
\text { ing the systems of care that physicians impact directly } \\
\text { Implement pre-printed fax-back forms to physicians that include all fields of information required } \\
\text { by physician, relevant to various clinical topics (e.g., pain), to enhance communication between } \\
\text { physician and nursing home staff } \\
\text { Involve local hospice to provide training on pain management to physicians and nursing home } \\
\text { staff }\end{array}$ \\
- & $\begin{array}{l}\text { Offer training session to medical directors on the role of the medical director in nursing home } \\
\text { quality improvement }\end{array}$ \\
- & $\begin{array}{l}\text { Write column in monthly newsletter to providers on the role of the medical director and } \\
\text { attending physicians in nursing home quality } \\
\text { Offer coaching to nursing home staff on how to give physicians the information they need to } \\
\text { make a decision }\end{array}$ \\
Partner with the local chapter of the American Medical Directors Association (AMDA) to advo- \\
cate for involvement in quality improvement among medical directors and physicians
\end{tabular}

Establishing a relationship with the State Survey Agency The QIOs in the six pilot states identified the State Survey Agency (SSA) as one of the most important and influential stakeholders with whom to establish a relationship. One reason for this is that nursing home providers often want to know if the SSA has been made aware of quality improvement material or recommended clinical interventions. It is also important to highlight for providers that the QIO has no regulatory responsibilities and is distinctly different from the SSA in its work with nursing homes.

As is true with any other partnership, regular communication is essential to success. QIOs found that they should regularly communicate to the SSA the kinds of quality improvement assistance they were planning to offer to nursing home providers, and if possible, obtain an endorsement of that assistance. An assurance of the State Survey Agency's awareness of quality improvement activities is essential in gaining nursing home providers' trust and in increasing their willingness to work with the QIO (or any other party), especially given the central role of the regulatory process in the current culture of nursing homes. Representatives from State Survey Agencies in most pilot states, such as surveyors, State MDS Coordinators, or department directors, participated on the steering committee of nursing home stakeholders, and made individual contact with QIOs during the pilot.

\section{Using the Quality Measures}

Nursing home providers need to understand the origin of the Quality Measures in order to make use of them in quality improvement. QIOs in the six pilot states offered educational workshops about the Quality Measures to all nursing homes in each state. The workshops focused on the Minimum Data Set (MDS) items that are the source of the Quality Measures, as well as methods used to risk ad- just the measures. While large workshops prove useful in introducing concepts to staff, ongoing consultation in smaller groups or one-on-one were most helpful in increasing understanding of the Quality Measures for nursing home staff. QIOs found that it was imperative to have an MDS expert (e.g., an MDS consultant or the state RAI coordinator) participate in the training sessions to clarify questions about appropriate coding for some of the MDS items, as there is tremendous variation in how nursing home staff code the MDS for the same MDS items.

\section{Engaging Physicians and the Medical Director in Quality Improvement}

Once QIOs in the pilot states began to provide quality improvement assistance to individual nursing home providers, they received requests for assistance in involving physicians in quality improvement efforts. Most of the requests came from nursing home administrators and directors of nursing.

The involvement of medical directors and physicians is vital in the attempt to change facility rates on certain Quality Measures, such as pain, which often depends on changes in prescription medication to improve pain management of residents. However, engaging physicians and medical directors to become directly involved in quality improvement projects at their facilities is often a challenge. Current regulations require that the medical directors participate in the facility's quality assurance committee, but the intensity of their involvement in quality improvement efforts is highly variable. Even when actively involved, most medical directors lack training in quality improvement processes. Historically, quality improvement has not been part of medical schools' curricula. QIOs found several strategies to overcome noninvolvement of the physician or medical director that 
Table 3: Practical Tips Shared by Nursing Home Provider Teams About Better Pain Management Practices

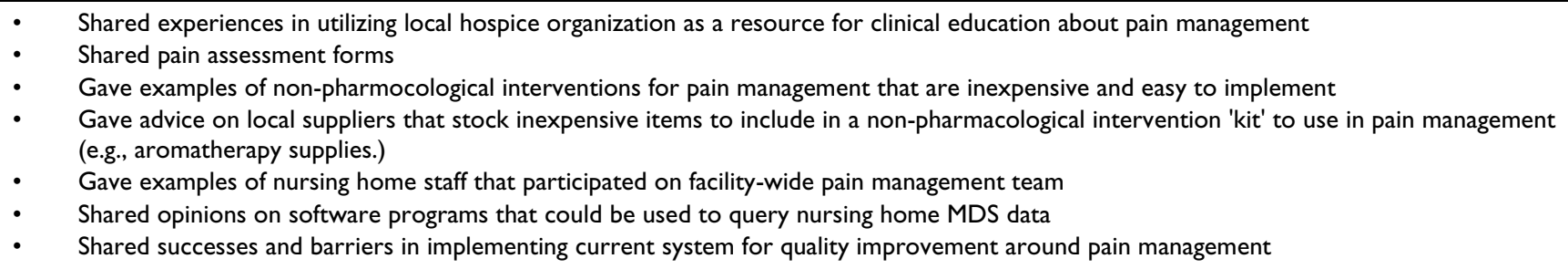

could be implemented by the QIO or the nursing home, as described in Table 2.

\section{Teaching Quality Improvement}

Teaching the principles of quality improvement to all nursing home staff, including direct care staff, is another common challenge to implementing quality improvement projects in nursing homes. Certified Nursing Assistants (CNAs) and State-tested Nursing Assistants (STNAs) provide the majority of direct patient care in nursing homes, but are often not included by the nursing home administration in quality improvement efforts. Moreover, quality improvement training is often presented at a college-education level, even though fewer than $5 \%$ of nursing assistants have had four or more years of college [7]. The lack of nursing assistants' involvement in quality improvement is unfortunate, as they are often best situated to contribute ideas to quality improvement efforts.

To reach all audiences in the nursing home setting, QIOs in the pilot states found that training to nursing home staff on quality improvement was most effective when presenters integrated the principles of rapid-cycle quality improvement into examples of small-scale improvements that could be made in care processes common to the nursing home setting. Quality improvement theory was not well understood by nursing home staff when general quality improvement concepts and terms (such as flowcharting and root-cause analysis) were presented without using nursing home care processes as examples, or were presented only with examples from manufacturing or other health care settings. QIOs were more successful when they used language common to the nursing home setting, rather than quality improvement jargon (i.e., PDSA cycles) in their training and communications. Relating quality improvement to the clinical method of careful observation, followed by intervention (i.e., some change in the care process), and then again careful observation to monitor the effect, was also well received by nursing home staff. Another successful approach is to teach one concept, such as data collection, at a time, allowing time in between for nursing home staff to apply the concept in either a mock workshop exercise, or as a real-life exercise at their own facility.

While one challenge in providing education on quality improvement principles is the content of the curriculum, another challenge consists of presenting information differently from the usual didactic seminars that nursing home staffs attend, which are not always effective. To overcome this barrier, the QIOs in the pilot states used both teleconferences and interactive sessions to facilitate groups of nursing home providers in sharing their experiences in quality improvement and in implementing changes in processes of care. For example, when QIO staff at these interactive sessions asked nursing home providers to explain in detail the change in process that they planned to make, other nursing homes in the group usually had experience in attempting the same change. It was apparent in the pilot that nursing homes have many practical tips to share about the effective application of widely accepted guidelines and protocols, and that the activity of sharing experiences is useful in inspiring and guiding nursing home teams in making changes to improve their processes of care. Examples of these practical tips are given in Table 3.

One barrier that nursing home teams face in doing quality improvement is lack of time for planning and evaluation. Thus, the QIOs found that some time at workshops was best allocated as time for nursing home teams to identify a change they could make in one process of care. QIOs then set a one-week deadline for nursing home teams to implement and measure a change they designed, in order to encourage teams to break down large systems changes into manageable steps. At subsequent workshops, QIOs facilitated productive discussions with nursing home staff to help them analyse of the results of the change, as measured by simple data collected by nursing home staff.

Finally, nursing home providers appreciated meetings or workshops that were arranged as a series, between which participants were assigned simple tasks relevant to what they had learned as "homework". Nursing home teams were then held accountable to present a report of the re- 
sults of their activities at the next meeting. When QIOs integrated nursing home providers' reports of their experiences into the workshops, the application of quality improvement strategies at participating facilities increased, because this reporting was valuable in identifying for all nursing home providers the tangible process changes that proved effective in improving care.

\section{Providing One-on-One Assistance to Nursing Homes}

QIO staff in the six pilot states that worked with nursing home providers on improving processes of care related to the Quality Measures found that nursing home teams were eager to begin a quality improvement effort in their facilities. Collaboration was most successful when QIO staff had nursing home experience themselves, and could lend a sympathetic and experienced ear to barriers that faced the team. The QIOs found that nursing home teams were more receptive to materials and advice when time was spent early in the project to assess the nursing home's team needs. This led to the development of detailed forms (or "checklists") that nursing home staff and QIO staff could use to assess whether or not recommended care processes for the clinical topic of focus were in place at the nursing home.

QIOs found that working with nursing home providers demands an approach that is as simple and straight-forward as possible. Data collection tools, for example, should be pared down to include only the information critical to the project. Rather than building a new team and a new set of inservices separate from what the nursing home already does, QIOs can coordinate with teams and meetings that already exist within the nursing home. QIO visits to nursing homes were more effective when an agenda was provided in advance, the meeting was limited to approximately one hour, and handouts were provided to focus the meeting.

\section{Convert Regulatory Compliance Culture to Quality Im- provement Culture}

Nursing home care is highly regulated by state and federal agencies. Failure to comply with regulations can result in stiff penalties, such as fines or exclusion from participation in Medicaid or Medicare reimbursement. Some nursing homes, particularly those seeking managed care and other insurance contracts, have begun to seek certification from the Joint Commission on Accreditation of Healthcare Organizations (JCAHO) for both long term and subacute care, which adds additional layers of requirements for the nursing homes. In addition, over $50 \%$ of nursing homes are part of a chain or are affiliated with one [8]. Corporate rules and protocols add another type of regulation with which nursing home staff must comply. Such externally imposed requirements, rules, or protocols often hinder the ability of nursing home internal quality im- provement teams to implement creative solutions that may lie outside of the established rule or protocol. For example, some chain organizations prohibit the use of "standing orders" in any form. Others require corporate pre-approval for changes in programs, for the sake of internal corporate consistency.

QIOs and other groups working in quality improvement need to acknowledge nursing home providers' requirements for regulatory adherence, but also must be aware of the regulations and clarify questions from nursing home staff about any perceived conflicts between regulations and quality improvement strategies (for examples of such conflicts, see Table 4). To assure quality improvement strategies are consistent with government regulations, frequent QIO consultation with the State Survey Agency is needed. Moreover, nursing homes staff may be more interested in quality improvement interventions that are related to salient regulations.

One of the greatest challenges to the national implementation of the Nursing Home Quality Initiative may be in changing the nursing home provider mindset from one of compliance with regulation through a system of quality assurance (focused on retroactively examining outcomes) to a mindset of quality improvement (focused on proactively improving processes and systems). For example, nursing home providers are accustomed to designing a "thirty-day plan of correction" in response to citations given by surveyors, in which the cited system of care is quickly revised to comply with the regulation. The plan of correction is then "re-inserted' into daily practice without any testing of the actual effect of the changes, but no proof of effectiveness is required. Quality improvement, however, is based on a process of making change iteratively, and demands testing prior to implementation to determine whether a change will actually result in an improvement.

Nursing home providers correctly perceive the government in an oversight role, enforcing regulations with punitive actions. Convincing nursing home providers that working with the QIOs on quality improvement initiatives is a voluntary, collaborative endeavor that is independent of the survey (inspection) process is a barrier that QIOs must overcome in order to be effective.

\section{Addressing Staffing Issues}

Turnover and vacancies make the formation and function of quality improvement teams more difficult, resulting in a low likelihood that any improvement will be adequately monitored or fully implemented. Workforce instability at the nursing home unavoidably impacts the relationship that the nursing home has with any outside agent, including the QIO. Even in the short duration of the pilot, QIO 
Table 4: Perceived Conflicts Between Regulations and Quality Improvement

- That in order to justify giving pain medication to a resident, the resident must be coded as having pain on the Minimum Data Set (MDS)
- That problems should be corrected in a short amount of time, rather than through small changes that lead to improvement
- That QIOs are doing research that requires permission from residents to participate
That identifying and documenting a problem that a facility team wants to improve will indicate to surveyors where the facility should be
cited for non-compliance with the regulations

staff witnessed turnover and vacancy in all positions. The challenge is not limited to working with ever-changing individuals on a quality improvement team, but also in accommodating a Quality Improvement Nurse or Director of Nursing who is covering a short-staffed unit rather than championing a quality improvement project. In one instance, a new administrator, uninformed about the pilot program and its objectives and methods, immediately withdrew the nursing home from the program, stating that it "was too time-consuming."

Having multiple contact persons at each nursing home helped the QIOs overcome a break in the nursing home's involvement. QIO staff developed relationships with the entire quality improvement team rather than just the Administrator or Director of Nursing. Given the Director of Nursing's many responsibilities, it is advised that someone other than him/her is designated as the quality improvement project's champion. Finally, frequent contact with all team members was necessary to be aware of and offset staff changes.

\section{Discussion}

Publicly reporting nursing home quality of care, and quality improvement projects in nursing homes, have existed independently of one another prior to this new federal initiative. For example, the Maryland Health Care Commission began reporting on a set of quality indicators for nursing homes in the state of Maryland in 2001 in response to a legislative mandate $[9,10]$. Additionally, the CMS Nursing Home Compare web site (at http:// www.medicare.gov) reported a set of quality indicators for all nursing homes nationally from 1998 to November 2002 , when the new set of quality measures became available. In both of the initiatives above, public reporting of nursing home quality indicators stood alone, without related support to nursing homes for quality improvement. The impact of public reporting on nursing home providers' interest in quality improvement has not previously been evaluated.

Prior nursing home quality improvement projects have been initiated in the absence of public reporting, by external groups such as the QIOs, State Survey Agencies, research groups, and nursing home corporations. Results indicate that these external groups have had success in improving processes of care (i.e., improved pain management, pressure sore prevention and treatment, and rates of influenza and pneumonia immunizations among nursing home residents [11].) CMS's Nursing Home Quality Initiative is a logical next step in creating a long-term commitment to quality improvement approaches driven by public reporting.

QIOs in the six pilot states found several key outcomes of the Nursing Home Quality Initiative that help to maximize the potential of public reporting to leverage effective improvement in nursing home quality of care. First, public reporting focuses the attention of all stakeholders in the nursing home industry on achieving good quality outcomes on a defined set of measures, and creates an incentive for partnership formation. Second, publicly reported quality measures motivate nursing home providers to improve in certain key clinical areas, and in particular to seek out new ways of changing processes of care, such as engaging physicians and the medical director more directly. Third, the lessons learned by QIOs in the pilot of this Initiative indicate that certain approaches to providing quality improvement assistance are key to guiding nursing home providers' desire and enthusiasm to improve towards a using a systematic approach to quality improvement.

\section{Conclusions}

The Nursing Home Quality Initiative has already demonstrated the potential of public reporting to foster collaboration and coordination among nursing home stakeholders and to heighten interest of nursing homes in quality improvement techniques. Through this Initiative, QIOs are identifying key lessons learned about approaches to providing quality improvement assistance to nursing home providers. The QIOs' work in this Nursing Home Quality Initiative will greatly contribute to the body of knowledge about facilitating quality improvement efforts in the nursing home setting, although it remains to be seen whether these efforts lead to improved quality of care in nursing homes nationwide.

\section{Competing interests}

None declared. 


\section{Authors' contributions}

SK contributed to the analysis of lessons learned and drafted the manuscript. DG conceived of the study, contributed to the analysis of lessons learned, and drafted the manuscript. PP conceived of the study, contributed to the analysis of lessons learned, and drafted the manuscript. GP, LP, LW, MF, ASP, and LB contributed lessons learned and their analysis. All authors read and approved the final manuscript.

\section{Abbreviations}

AMDA - American Medical Directors Associations

CMS - Centers for Medicare \& Medicaid Services

CNA - Certified Nursing Assistant

JCAHO - Joint Commission on Accreditation of Healthcare Organizations

MDS - Minimum Data Set

PDSA - Plan Do Study Act

QIO - Quality Improvement Organization

RAI - Resident Assessment Instrument

SSA - State Survey Agency

STNA - State-tested Nursing Assistant

\section{Acknowledgements}

The analyses upon which this publication is based were performed under a subcontract entitled "Six-State Pilot for Release and Publication of CMS Quality Indicator (QI) Measures and Testing of QIO Intervention to Improve Care Related to QI Measures" from the lowa Foundation for Medical Care and sponsored by the Centers for Medicare \& Medicaid Services (CMS, formerly the Health Care Financing Administration), Department of Health and Human Services. The content of this publication does not necessarily reflect the views or policies of the Department of Health and $\mathrm{Hu}$ man Services, nor does mention of trade names, commercial products, or organizations imply endorsement by the U.S. Government. The authors assume full responsibility for the accuracy and completeness of the ideas presented. This article is a direct result of the Health Care Quality Improvement Program initiated by CMS, which has encouraged identification of quality improvement projects derived from analyses of patterns of care, and therefore required no special funding on the part of this Contractor. Ideas and contributions to the author concerning experience in engaging with issues presented are welcomed.

The authors wish to thank Rosa Baier and Melissa Miranda for their research and administrative support in writing this paper.

The authors of this manuscript would like to dedicate this work to the memory of Peggy Parks, whose energy, innovation and dedication to the Nursing Home Quality Initiative in both the pilot phase and national implementation contributed greatly to its development.

\section{References}

I. Lansky D Improving quality through public disclosure of performance information Health Affairs 2002, 21:52-62

2. Marshall MN, Shekelle PG, Leatherman S and Book RH The public release of performance data: What do we expect to gain? A review of the evidence JAMA 2000, 283: | 866- |874

3. Chassin MR Achieving and sustaining improved quality: lessons from New York State and cardiac surgery Health Affairs 2002, $21: 40-51$

4. Kizer KW Establishing health care performance standards in an era of consumerism JAMA 200I, 286:1213-1217

5. General Accounting Office (GAO) Nursing Homes: Public Reporting of Quality Indicators Has Merit, but National Implementation Is Premature GAO-03-187 2002,

6. "Report on Evaluation Activities for the Nursing Home Quality Initiative Pilot." Centers for Medicare \& Medicaid Services 2003, [http://www.cms.hhs.gov/providers/nursinghomes/nhi/ Report20021017.pdf]

7. Yamada Y Profile of home care aides, nursing home aides, and hospital aides: historical changes and data recommendations Gerontologist 2002, 42:199-206

8. Jones A The National Nursing Home Survey: 1999 summary National Center for Health Statistics. Vital Health Stat 2002, I3(I 52):

9. Wright R, Patry G, Mattke $S$ and Gifford DR Public reporting of nursing home quality of care [abstract] J Am Geriatr Soc 2002 , 50(suppI):SI72-SI73

10. "Maryland Nursing Home Performance Evaluation Guide" Maryland Health Care Commission 2002, [http://www.mhcc.state.md.us]

II. Gifford DR, Teno JM, Patry G, Banks S and DeSilva D Improving pain management in nursing homes [abstract] J Am Geriatr Soc 2002, 50(suppl):

\section{Pre-publication history}

The pre-publication history for this paper can be accessed here:

http://www.biomedcentral.com/1471-2318/3/2/prepub
Publish with Biomed Central and every scientist can read your work free of charge

"BioMed Central will be the most significant development for disseminating the results of biomedical research in our lifetime. " Sir Paul Nurse, Cancer Research UK

Your research papers will be:

- available free of charge to the entire biomedical community

- peer reviewed and published immediately upon acceptance

- cited in PubMed and archived on PubMed Central

- yours - you keep the copyright
BioMedcentral 